\title{
Intern Designed Modules for Conducting Potential Microgravity Solidification Experiments aboard the International Space Station \\ NASA Internship Report
}

\author{
Justin McElderry ${ }^{1}$, Andrew Bouchard ${ }^{2}$, and Tristan Phillips ${ }^{3}$ \\ ${ }^{1}$ Student (Department of Mechanical Engineering, Milwaukee School of Engineering) \\ Milwaukee, WI; ${ }^{2}$ Student (Department of Mechanical Engineering, Merrimack College) North \\ Andover, MA; ${ }^{3}$ Student (Department of Electrical and Computer Engineering, University of \\ Alabama) Tuscaloosa, $A L^{*}$
}

\begin{abstract}
MMaJIC (Microgravity Materials Joining Investigation Chamber) is a modular experiment chamber for performing materials science investigations. MMaJIC provides a controlled and sealed test environment for a wide variety of soldering and brazing experiments to examine porosity in microgravity. MMaJIC's modular cartridge design includes a tray for housing the solder experiments and demonstrates simplicity to astronauts. SoLIDD (Solid Liquid Interface Directional Device) is a device for conducting directional solidification experiments which enables control of the microstructural development. SoLIDD has capability for varying temperature gradient and growth velocity. These quantities use heaters, coolers, and a directional drive unit. ICED-T (Interface Control Experiment with Directional Translation) is an apparatus that conducts directional solidification experiments of transparent materials which allows direct observation of the solid/liquid interface. Currently, it is in a breadboard phase that consists of the components mounted to a surface that allows the sample to be placed in an upright position. Orientation of the experiment (solid on the bottom, liquid on top) is important during testing ground samples to maintain stability. Ultimately, the goal of ICED-T is to conduct successful ground tests and then be configured to fit into an enclosure similar to MMaJIC and SoLIDD that could be used aboard the ISS.
\end{abstract}

\section{Introduction}

Gravity-based phenomena drive all ground-based melting and solidification processes. Buoyancy leads to convection and tends to sort layers of a fluid by mass. Fluid shapes are a balance of surface tension, wetting and gravity, and gravity-driven settling is often observed. In microgravity, secondary terms such as thermocapillary-driven convection take a primary role in governing microstructural development. Since these processes are fundamental to routine manufacturing and repair methods such as soldering or brazing, a better understanding of melting and solidification in microgravity is essential to any Long-duration exploration efforts. Previous studies of in-space

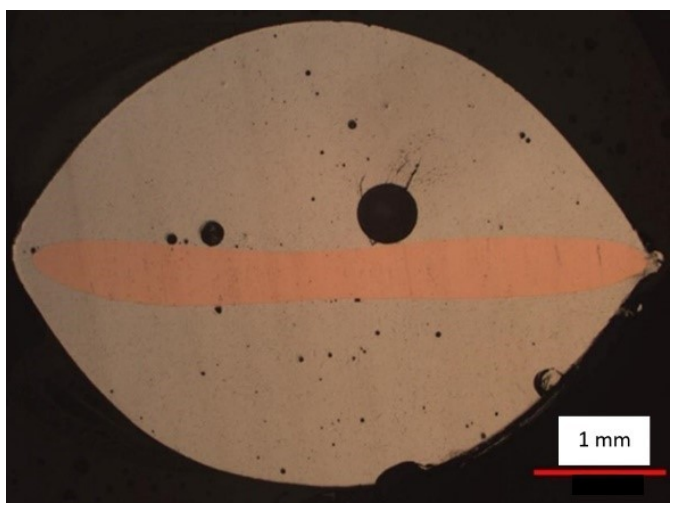

Figure 1: Flux bubbles (porosity) are seen at the solder/copper wire (joint) interface from the ISSI samples. soldering have shown that normal ground techniques result in joints that have higher porosity, reduced structural integrity, and suboptimal electrical and thermal conductivity at a solder joint 
* Experiment support offered by the NASA Marshall Space Flight Center Engineering Materials Section, and Wisconsin Space Grant Consortium

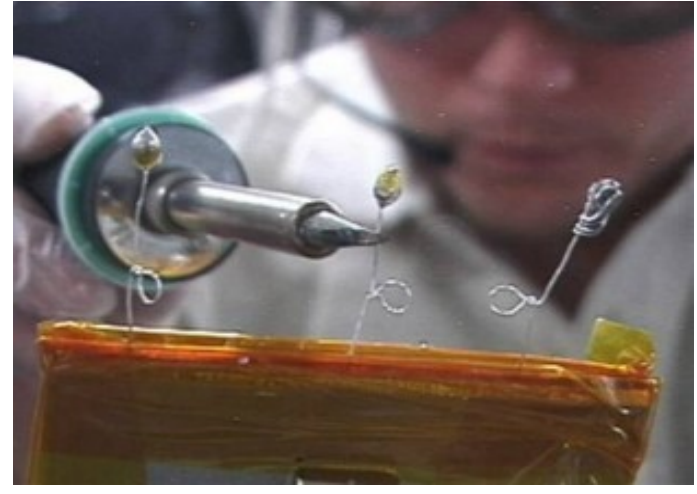

Figure 2: Photograph of ISS Science Officer, Mike Fincke, conducting a set of melting experiments within the Maintenance Work Area shown at a microscopic view in Figure 1. Further studies of solid/liquid interfaces and phase changes in microgravity are needed to solve problems in applications like these (Daly 2017).

To meet the need for more studies, the Microgravity Materials Joining Investigative Chamber (MMaJIC) was proposed for flight on the International Space Station (ISS) and was designed as a flexible platform for processing a wide variety of material science payloads. Each sample was prepared in advance on the ground, fitting into a sample tray securely that is inserted into the on-orbit housing. Parameters for each experiment were encoded into an on-cartridge SD card, which was then read by MMaJIC's microcontroller and used to control the voltage supplied to three power lines, which were primarily used to control the temperature of heating elements along a specified thermal profile. Built-in thermocouples recorded temperature from each sample and provided the feedback needed for control. These data were logged to the built-in SD card and sent back with the processed sample. The entire operation requires minimal crew input, using a one-button interface to start each sample. MMaJIC provides an environment to perform high-quality science, with minimal operation time and high sample throughput for future research.

The objective of Solid Liquid Interface Directional Device (SoLIDD) is to directionally solidify lower temperature metals and alloys. SoLIDD uses both a heater and an electric cooler which allows for accurate control of the thermal gradient across the material's solid/liquid interface within the Sample Ampoule Assembly (SAA). With control of the gradient, the SAA is translated at a controlled rate so the resultant microstructure can be characterized in terms of the processing parameters. ICED-T complements the Pore Formation and Mobility Investigation apparatus, which used real-time imaging and control thus allowing investigators to examine the dynamics of solidification in microgravity via ISS. downlink, Figure 3 (Spivey 2003). The ICED-T apparatus was designed to use the Maintenance Work Area (MWA) aboard the ISS for quick accessibility.

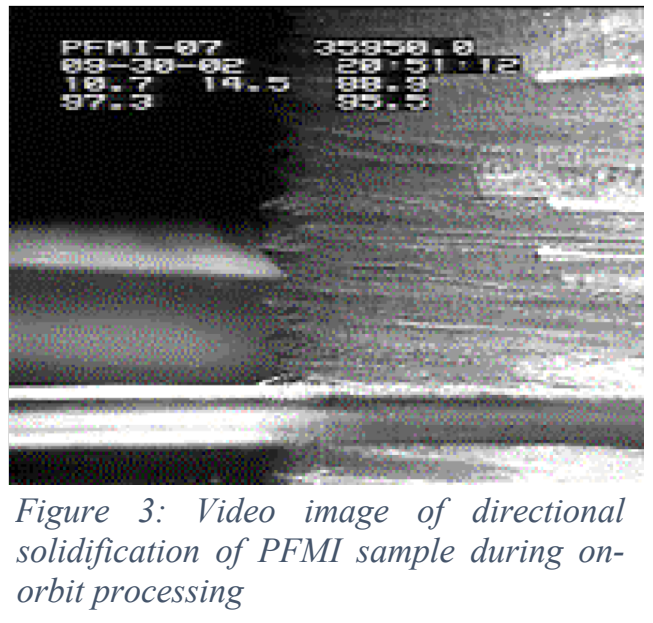

orbit processing 


\section{Materials and Methods}

MMaJIC went through a second iteration design and development in the summer of 2018 resulting in upgrades which opened new experimental opportunities. SoLIDD underwent a second iteration in the summer of 2019 which included a geared directional drive unit, more space for electronics, a bigger viewing window, and a hinged door for inserting sample tubes. ICED-T, following SoLIDD, was designed and built to provide a ground unit for scientists to conduct directional solidification experiments with transparent liquids.

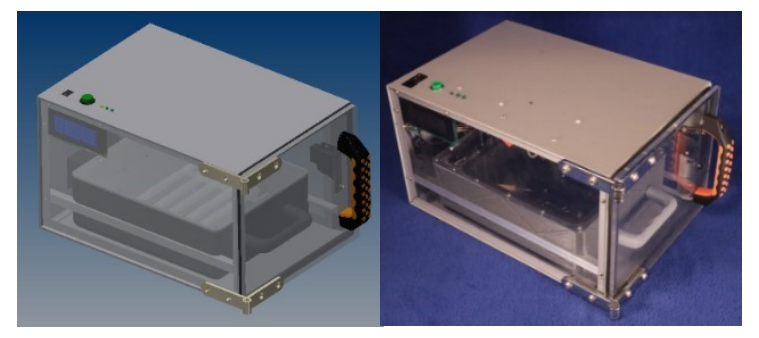

Figure 4: CAD model of MMaJIC (left) and final model of MMaJIC (right).

The mechanical design of the housing unit for MMaJIC remained the same as shown in Figure 4. A new "smart" door latch was installed to sense if the door was open prior to experimentation. The overall size of MMaJIC remained 10.19" wide, 8 " high, and 14.88 " long to easily fit within the MWA as well as provide two levels of containment.

Sample trays acquainted with the unit and are presently 12.4" in length (with the handle), 8.35" in width, and 3.4" high with more volume added for critical electronics. Trays were 3D printed and laser-cut to facilitate experiments and, where possible, used space-flight approved materials. The sample trays and the unit of MMaJIC followed as many ISS design standards possible. The trays are also extremely customizable with modular CAD files such that principal investigators can easily accommodate their experiments. Three sample trays were produced to demonstrate the flexibility and wide range of experiments that are possible with MMaJIC.

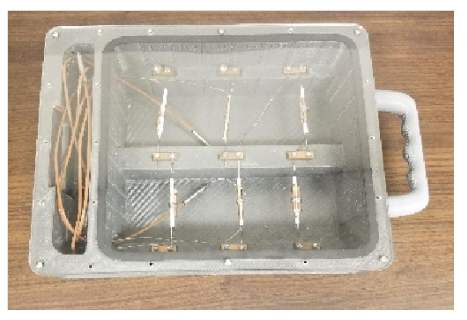

Figure 5: MMaJIC soldering tray capable of holding six soldering tests

The soldering tray, shown in Figure 5, enabled testing of six uniquely designed samples. The solder samples were wrapped around a small heater wire secured by screws; thermocouples controlled and monitored the temperature of the samples.

The solidification sample tray shown in Figure 6 contained six sample tubes for solidification experiments. Individual heating coils were wrapped around the quartz tubes which held a lead-tin alloy and secured by vibration resistant silicone rubber clamps designed to easily exchange samples. The heating wires are connected to terminals on the bottom of the tray and thermocouples controlled and monitored inside the tube. After processing the samples can be easily removed from the tray for subsequent evaluation.

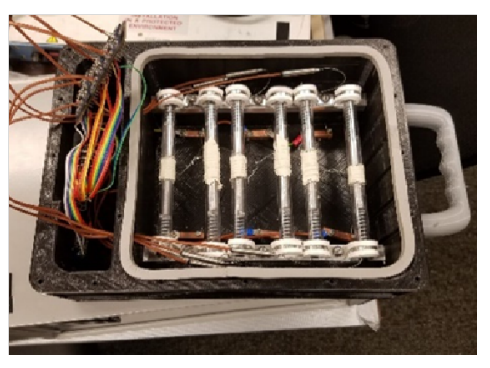

Figure 6: MMaJIC solidification tray capable of holding six quartz tubes 


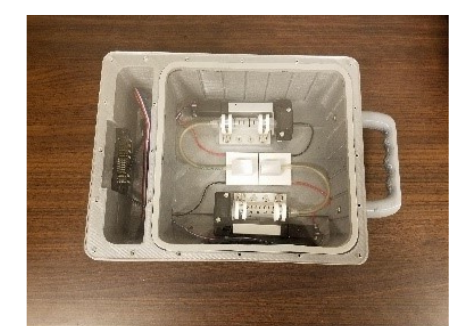

Figure 7: MMaJIC fluids tray with two syringes for fluid suspension experiements

The sample tray shown in Figure 7 was a demonstration unit that housed two syringes, each connected with a tube for fluid transport; two small thermoelectric heaters/coolers are also included to show additional versatility. The syringes can be plunged by actuators on each side that can provide $5 \mathrm{~N}$ of force to viscous and non-viscous fluids at relatively slow velocities $(10 \mathrm{~mm} / \mathrm{s})$. This sample tray, for example, allows scientists to examine the behavior of mixed fluid suspensions in microgravity.

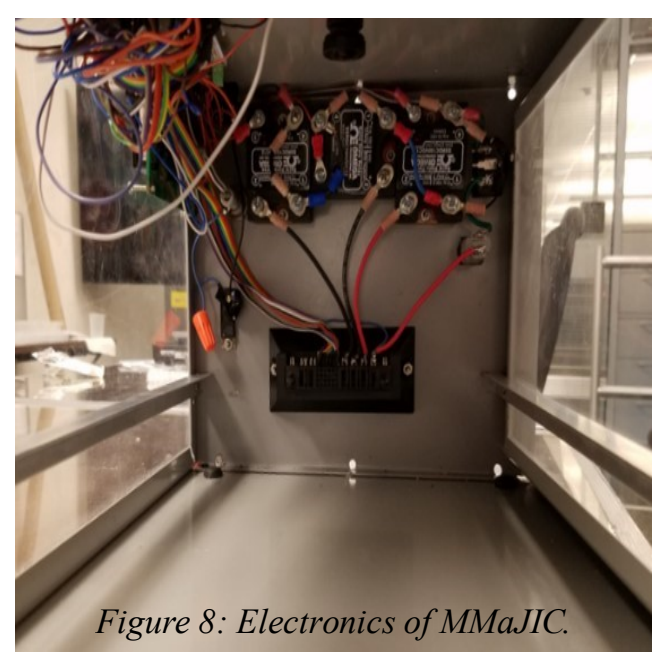

MMaJIC's electronics are designed to be adaptable and provide an environment to perform many different types of experiments. The unit can supply variable DC voltages from 0 to 30 volts, with a maximum allowed current of $10 \mathrm{~A}$ to components, Figure 8 . Since each new tray changes the equivalent resistance of the unit, the current required at a given voltage will also be tray dependent. The electrical design provides a great amount of tolerance thus allowing different experiments to be run. These design considerations satisfy the requirements for the Plug and Play Plan aboard the ISS, with MMaJIC receiving power from a Utility Outlet Power (UOP) unit near the MWA.

After the tray is inserted initiation of the experiment is controlled with one button. All the safety monitoring, heating control and data collection is fully automated by the onboard microcontroller, while an LCD screen provides all vital information to the operator/user. Circuit protection included placing fuses in an easy to access centralized location on the box's housing with a 10A fuse to prevent the unit from drawing too much power from the UOP and a $0.5 \mathrm{~A}$ fuse to protect the microprocessor and I/O boards from unforeseen current spikes. Gains and parameters were adjusted this year. The microcontroller continually monitored temperature and debris particle sensors from the sample for potential errors and auto shutdown conditions. There are times when the controller sends interfered temperature data values (most being $0^{\circ} \mathrm{C}$ ) when it starts controlling the temperature. A filter was implemented to eliminate the interfered values so that MMaJIC would not auto shutdown. This filtering process also eliminated individual temperature readings deemed not physically possible during the experiment, i.e., when a reading was less than $20 \%$ of original programed temperature value. This is a method used on the ISS when radiation affects data. 


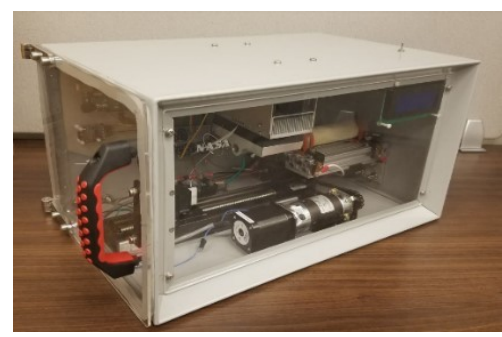

Figure 9: Final assembly of SoLIDD
SoLIDD was designed to conduct directional solidification experiments safely and efficiently with the intent of examining a broad range of lower melting temperature $\left(<300^{\circ} \mathrm{C}\right)$ metals and alloys. SoLIDD received several upgrades during the summer of 2019. These included electronics and a new gearbox capable of lower directional solidification speeds $(<1 \mathrm{micron} / \mathrm{sec})$; an easier sample interface for users was also incorporated. SoLIDD has five main mechanical systems: the housing unit, cooler, heater, directional drive unit (DDU), and sample ampoule assembly (SAA), Figure 9.

The housing unit of SoLIDD expanded out from 15" x 7" x 9" to 17" x 11 " x 9.5", allowing for a more organized set up of mechanical/electrical components and the addition of a gearbox. An 11" $x$ 9.5" hinged door with a gasket was placed on the side of the unit to facilitate accessing the sample. It takes $5 \mathrm{lbs}$ of force to pull and open the door, which follows the ISS guidelines (NASA). An LCD screen was attached to the front viewing window that provides feedback to the user on temperature data as well as progress updates on the experiment. The thermoelectric cooling unit remained the same (TE-Tech CP-040HT) and provided temperatures between $-18^{\circ} \mathrm{C}$ and ambient. Milled brackets were attached to the cooler to optimize heat transfer to the SAA after last year's thermal analysis (McElderry). Carbon fiber felt was placed inside the bracket to maximum contact and increase heat transfer. The 6 " heater unit adjacent to the cooler was remodeled using 2 layers of alumina tubing with an internal heater coil (kanthal wiring) capable of $300^{\circ} \mathrm{C}$. The heater was secured on an adjustable platform by two

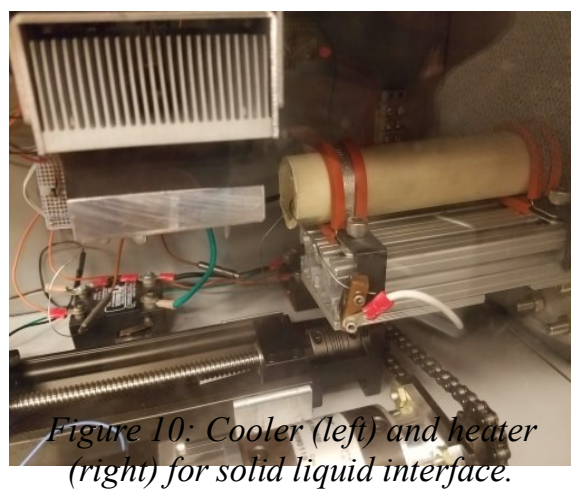
heat-resistant clamps. It can be adjusted by $1 "(2.54 \mathrm{~cm})$ to allow variance in the temperature gradient between the cooler and heater, Figure 10.

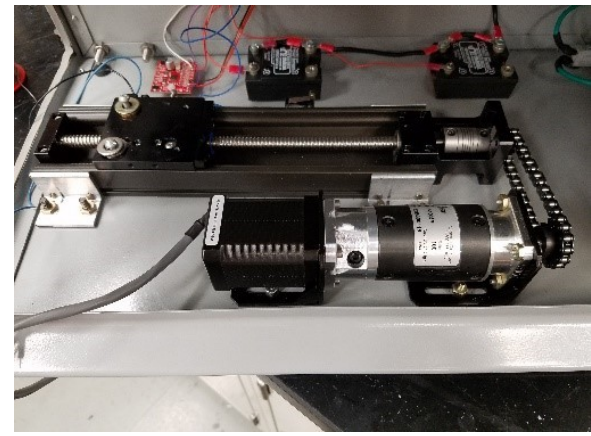

Figure 11: Directional drive unit in SoLIDD
The directional drive unit (DDU) consisted of a linear actuator slide, SAA mount, NEMA 17 stepper motor, 100:1 gearbox, and a sprocket/chain assembly, Figure 11. To ensure the desired translation rates $(<1 \mathrm{micron} / \mathrm{second})$ can be met a 100:1 gearbox was attached to the original stepper motor. To conserve space, a sprocket and chain assembly (13:9 sprocket ratio) was incorporated to drive the linear actuator slide which subsequently moves the SAA. When the SAA is in place, the user would close the hinged-door and initiate the experiment. The test SAA consisted of a clear quartz tube, held by an aluminum flange, which contained the sample alloy. 
The summer of 2019, the electrical design of SoLIDD was reworked to be simpler and more reliable. By reusing the temperature control system from the previously mentioned MMaJIC unit, most of SoLIDD's electrical architecture could remain unchanged allowing for rapid development and construction of the unit. How SoLIDD differs from MMaJIC is its use of a linear actuator, and this created a unique challenge for the electrical and software teams, Figure 12. Since both the active temperature control and the stepping of the motor must be performed in parallel to maintain high control resolution. in both systems, a second microcontroller was implemented. By including two separate processors, these

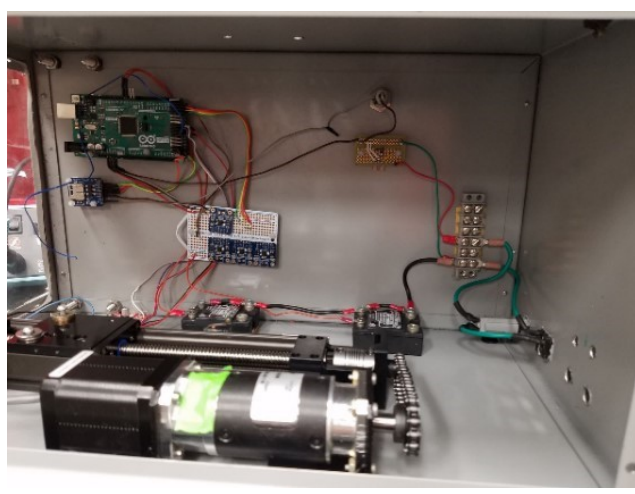

Figure 12: Electronics assembly for SoLIDD two computations could be performed simultaneously without impacting one another.

ICED-T, Figure 13, is a new experimental module intended to directionally solidify transparent materials. This experiment allows examination of dendrite formation and solid-liquid interfaces

Directional solidification on Earth is best conducted vertically, with the hot zone upwards to minimize convection effects. Similar to SoLIDD, there needed to be approximately 1" of space for viewing. To facilitate viewing of ice/water interfaces, two coolers were installed instead of a heater and cooler because liquid samples are being tested and do not have a high melting point.

Directional translation speeds from 0.2 to 10 microns/second are possible.

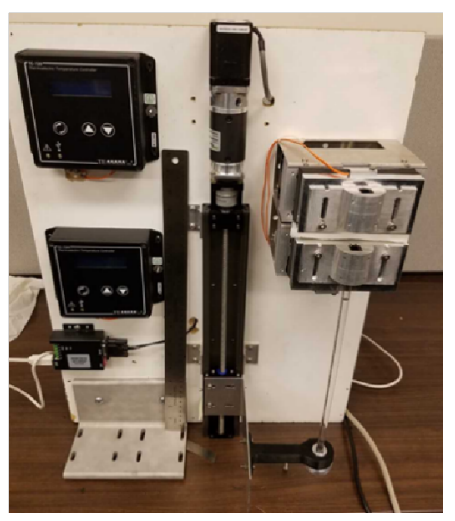

Figure 14: Final assembly of ICED-T
The directional drive unit (DDU) on ICED-T contained a linear slide actuator with a 0.2" pitch, a PGCN17-1005M 100:1 gearbox, and an STP-MTR-17060 NEMA 17 stepper motor connected together as shown in Figure 14. Connected to the DDU was the sample ampoule assembly (SAA) that translates between the coolers.

The two coolers were TE-Tech CP-040HT thermoelectric devices to provide test temperatures between $-18^{\circ} \mathrm{C}$ and ambient temperatures to develop solid/liquid interfaces for examination. Milled brackets were attached to the coolers with carbon fiber felt inside to increase thermal conductivity to the SAA. Connected to the coolers were the controllers that allowed the user to adjust temperatures to establish the solid/liquid

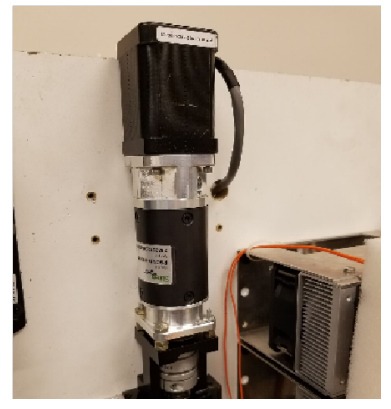

Figure 13: Stepper Motor and gearbox used for directional solidification interface. The DDU translation rate was controlled by a computer with software from the vendor, Figure 15. The user inputs the desired travel distance and makes the speed of the 
motor (fixed $0.0042 \mathrm{rev} / \mathrm{sec}$ ) and the gearbox ratio can reduce this speed even more by a factor of 100 .

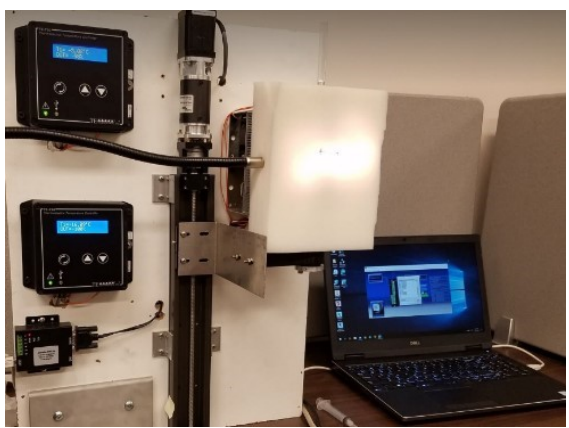

Figure 15: User Interface of ICED-T with cooler controllers and software on a laptop to control translation speed
The electronics systems for ICED-T are mainly comprised of two power supplies that deliver power to the coolers, motor, advanced micro-stepping drive, serial to USB adapter, and the two temperature controllers seen in Figure 16. The first power supply is a PS-24-6.5A unit supplied by TE Technology that provides 24 volts DC and up to $6.5 \mathrm{~A}$. Power from the unit is directed to the two temperature controllers that are connected to the thermoelectric coolers, supplying them with power from the PS-24-6.5A unit. The second power supply is an STPPWR-3204 unit that can supply 32 volts DC at 4 A supplied by Automation Direct providing power to the motor.

Temperature controllers for ICED-T were both supplied by TE Technology defined as the TC-720 unit that is capable of both heating and cooling and can control up to 36 volts and $20 \mathrm{~A}$ for each thermoelectric. The controllers read temperature data from MP-3193 NTC thermistors that have a theoretical measuring range from -20 to $100^{\circ} \mathrm{C}$ and provide accurate temperature data for precision control. Establishing parameters on the controller is simplified by the LCD display and buttons attached to the casing which allowed for quick navigation. TC-720 controllers recognized custom temperature profile programs that can be installed from a computer into the controller.

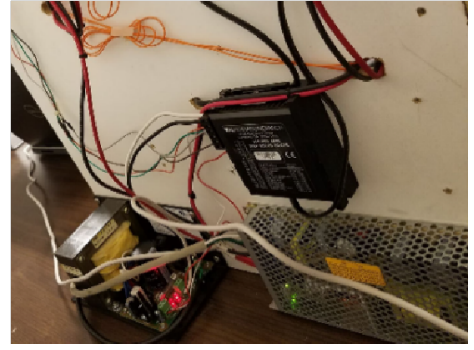

Figure 16: Power Supply needed for operating experiment on the other side of particle board
Motor control was accomplished using the STP-DRV-4850 advanced micro-stepping drive supplied by Automation Direct and communicates to a computer through a serial to USB adapter. Software was supplied by Automation Direct which allows for commands to be sent to the drive through a terminal for control of speed, acceleration, step count, and many other commands. The connection between the motor and micro-stepping drive was done through a 4-pin connector that defined each of the motor phases and had to be aligned to the pins that would read them on the drive.

\section{Key Results}

Several modifications were adapted into the current MMaJIC unit to improve experiment and engineering performance of the apparatus and sample trays to develop a ground unit that resembled space-flight readiness. One goal of MMaJIC was to reduce audible noise. The frequency being emitted from the unit was $500 \mathrm{~Hz}$, and it was boosted to $30,000 \mathrm{~Hz}$, resulting in silence to an average human being.

Sample trays were 3D printed and covers laser-cut in under two days. The trays were composed of space-flight approved polycarbonate that took up to 42 hours to print. The trays were 3D printed on a 12" x 12" x 6" heated bed $\left(90^{\circ} \mathrm{C}\right)$ through the Lulzbot Taz 6 using $750 \mathrm{~g}$ of PC-Max (tray 
and handle) with a $10 \%$ infill. Tray lids were made from a 24" x 12 " laser-cutter with EMI resistant polycarbonate to be converted to space-flight hardware. Modifying the tray in CAD makes it easily modular to PIs, universities, and other researchers interested in running their experiment with MMaJIC as shown in Figure 17.
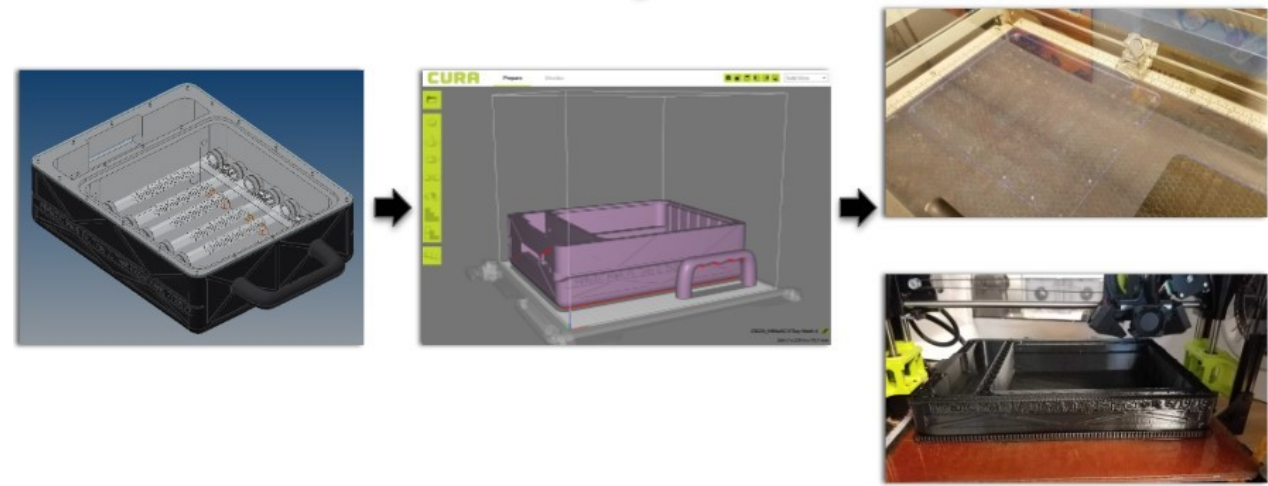

Figure 17: Manufacturing process of sample trays.

Sample trays provide a strong and vibration-resistant containment for the samples to be processed.

The sample tubes were tested at $300^{\circ} \mathrm{C}$, and the end of the tubes were warm (roughly $40^{\circ} \mathrm{C}$ ). Compared to thermal steady-state FEA modeled in Figure 18, the results were reasonably accurate when compared to the physical temperature. This investigation verified that silicone rubber is a plausible insulator for securing the ends of the sample tubes.

With the method of using clamps and aluminum bars to

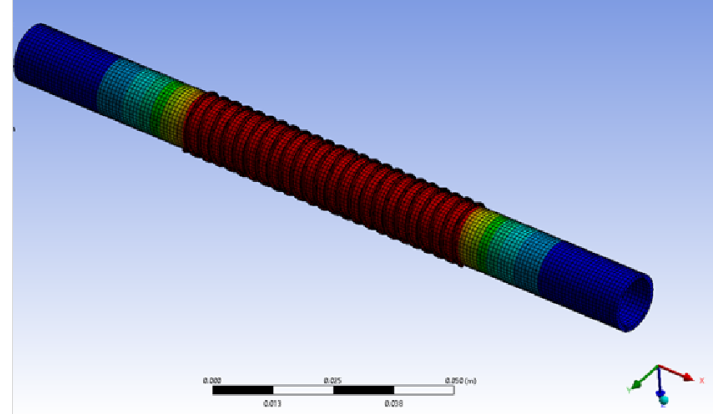
support the sample, there is room to expand trays and optimize the number of samples. Quartz sample tube lengths were 6" as shown in Figure 19. The result from a similar experiment was replicated where lead $30 \%$ and $40 \%$ volume tin samples were heated to and held at $204^{\circ} \mathrm{C}$. The problem with testing on the ground vs. testing in microgravity is that the lighter tin dendrites float up due to buoyancy as shown in Figure 19.

Figure 18: Thermal analysis for sample tubes

(Red: kanthal wire at $300^{\circ} \mathrm{C}$; Blue: alumina tube at $22^{\circ} \mathrm{C}$ ).

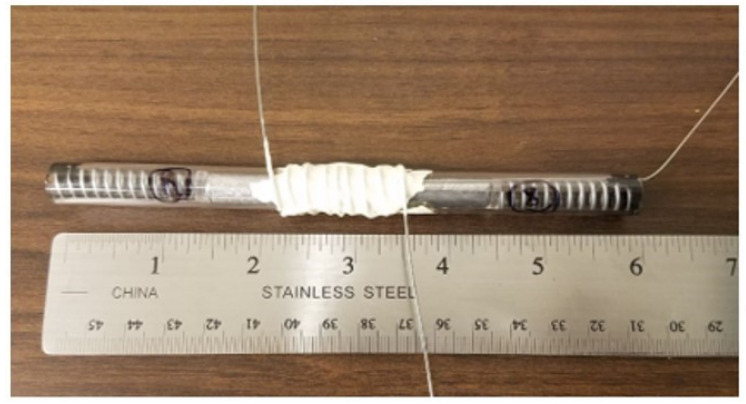

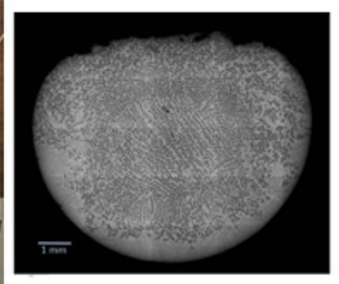

40 volume $\% \mathrm{Sn}$

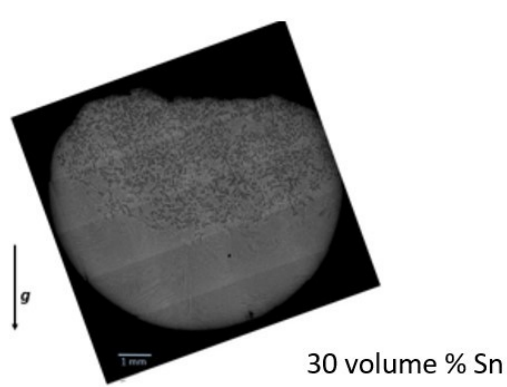

30 volume $\% \mathrm{Sn}$

Figure 19: Kanthal wire wrapped around the outside of the tube for resistance heating and ceramic cement coated around the wires (left) and gravity effects on microstructure during holding 1) slumping of the sample and 2) Segregation (buoyancy) of tin dendrites (right). 
With the samples from MMaJIC, $\mathrm{Pb}-6 \mathrm{wt} . \% \mathrm{Sb}$ alloys were cast into quartz tubes containing a k-type thermocouple. The sample tubes were heated to $340^{\circ} \mathrm{C}$ and $330^{\circ} \mathrm{C}$ which melted the alloy. The samples were then cooled down, allowing the alloys to solidify inward from each end resulting, eventually, in a central region of equiaxed growth as shown in Figure 20.
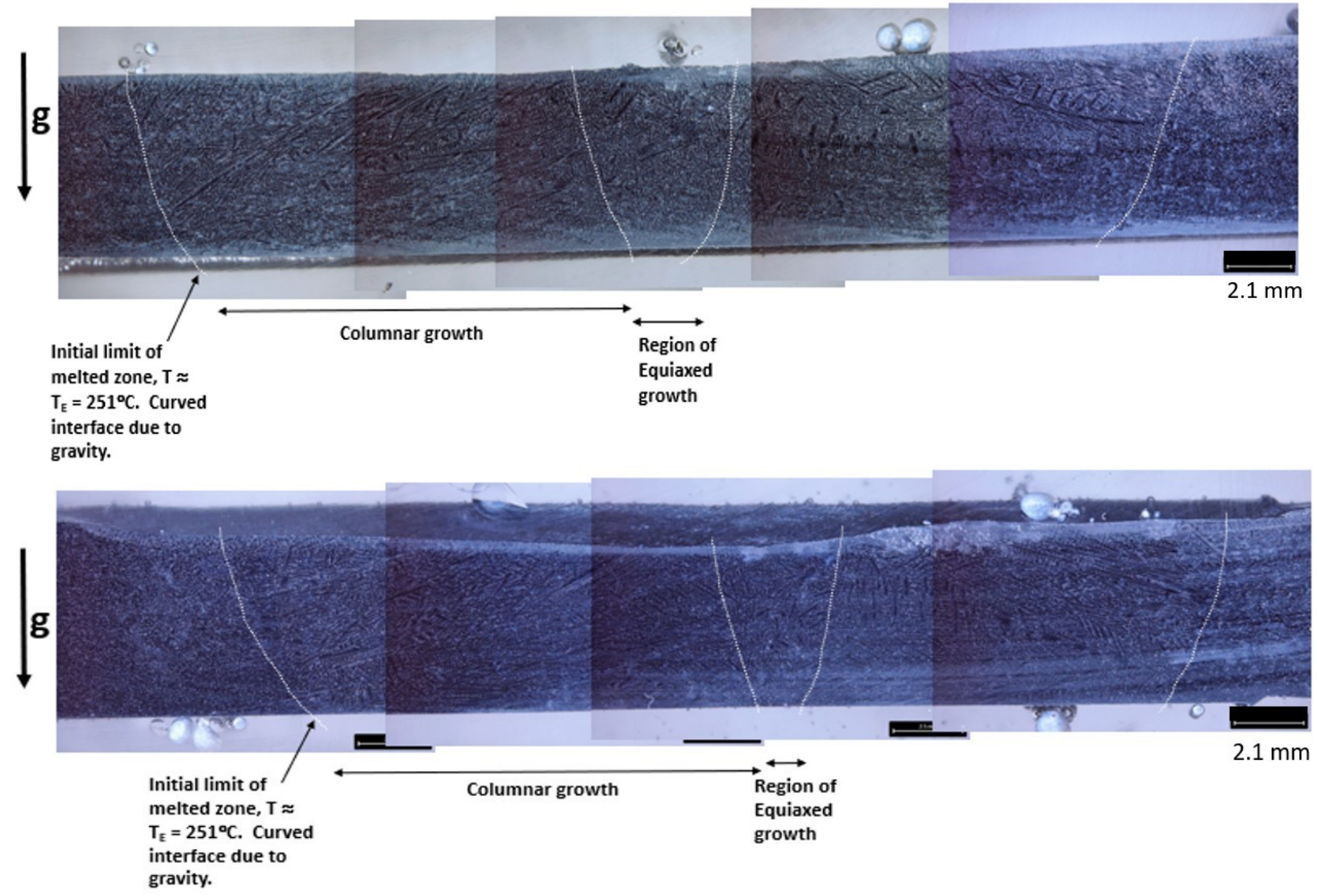

Figure 20: Two Pb-6 wt.\% Sb alloys were cast into quartz tubes to view the microstructure during cooling.

After the experiment, MMaJIC temperature data stored on an SD card that showed the controlled heating and cooling rates of the $\mathrm{Pb} \mathrm{Sb}$ alloys tested in Figure 21. Here electromotive forces (EMF, potential difference in charge) caused skewed and not physical temperature values to be recorded.
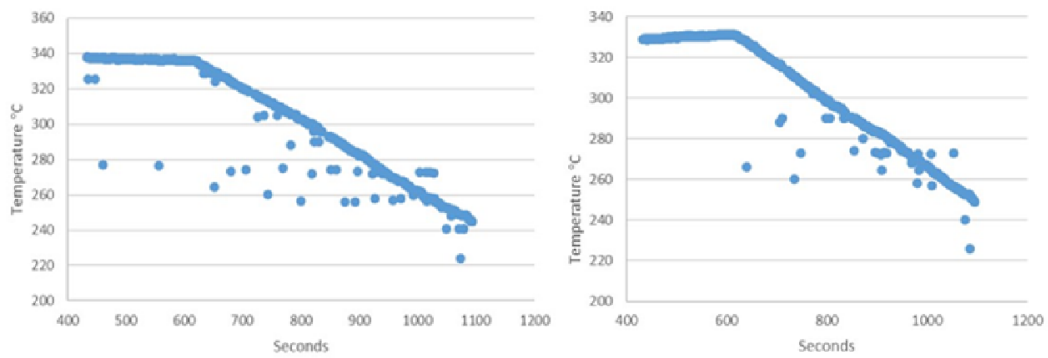

Figure 21: Heating control and cooling temperatures for two Pb-6 wt.\% Sb alloys processed in MMaJIC. Top sample from Figure 20 (left) and bottom sample from Figure 20 (right).

Another objective this summer was to eliminate these false temperature readings, which is not uncommon on the ISS. MMaJIC was programmed to eliminate datum points that were $20 \%$ off 
from the preceding temperature reading; this resulted in a much smoother plot from filtering junk points out with a different trial, Figure 22.

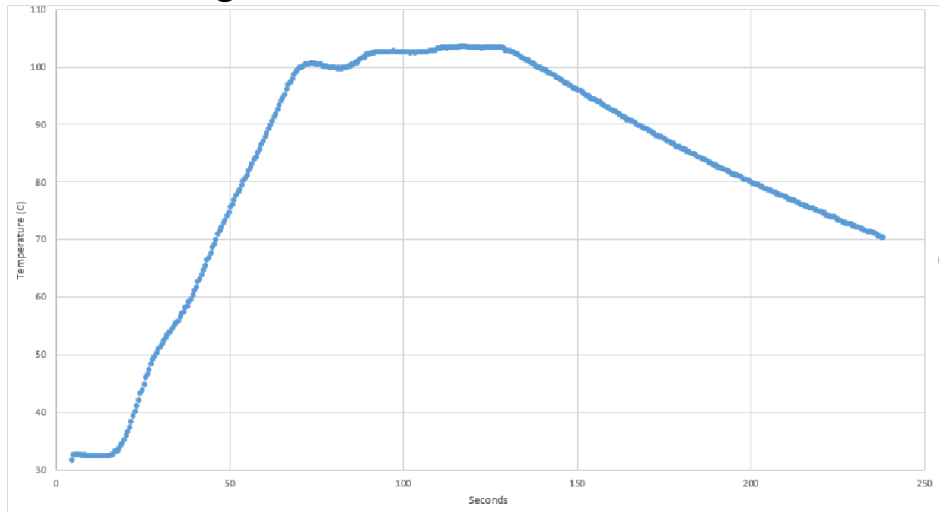

Figure 22: EMF filtered temperature readings with controlled heating and cooling.

For viewing sample processing, a 1080p 30fps fisheye camera was placed directly above the sample tray, which sent video to the SD for later viewing by the investigator, Figure 23 (left). Figure 23 (right) shows Kanthal wire wrapped around a quartz sample tube containing a Tin-Lead alloy that was later melted.

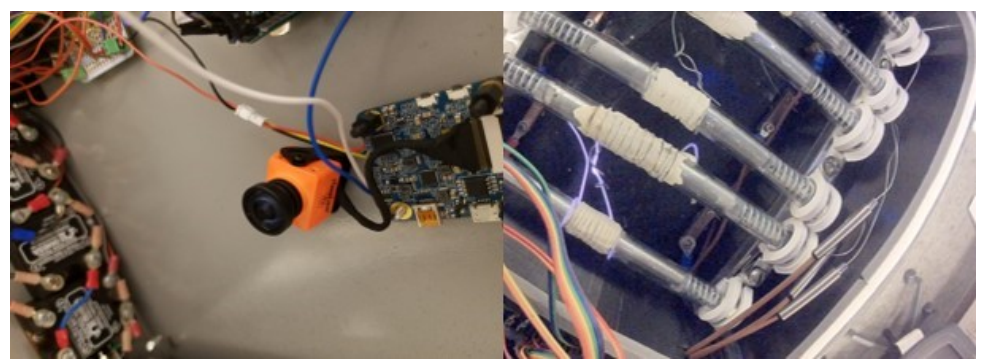

Figure 23: Camera mount on ceiling of MMaJIC (left) viewing the heated kanthal wiring (right)

SoLIDD was designed to reach temperatures up to $300^{\circ} \mathrm{C}$. Translation speeds could not be tested accurately in SoLIDD due to the controller's current limitations. Here the motor/gearbox assembly was the same as used in ICED-T so directional solidification speeds can easily be determined when a newly ordered controller is installed.

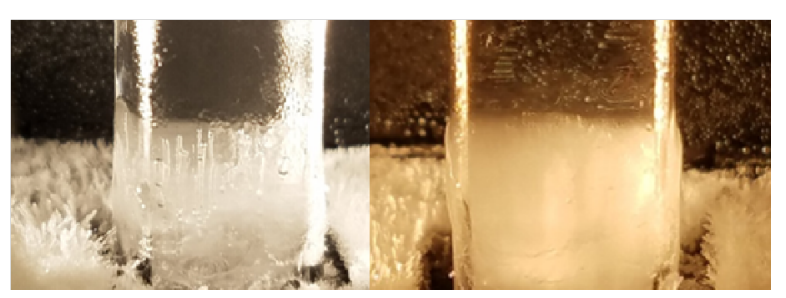

Figure 24: Solid/liquid interface and directional solidification of water with dissolved air in the ice (left) and trehalose solution (right)
In ICED-T, the directional drive unit demonstrated a minimum speed of $0.196 \mathrm{microns} / \mathrm{sec}$. The two identical cooling units were able to reach $-18.0^{\circ} \mathrm{C}$. Water and trehalose (5\% sugar water) samples were tested, primarily to see if a viewable solid/liquid interface could be established prior to initiating directional solidification. This was achieved in both samples. Water was tested first with a setting of $0^{\circ} \mathrm{C}$ for the top cooler, and $-18^{\circ} \mathrm{C}$ on the bottom one with the successful result shown in Figure 24. For the trehalose solution, the top cooler was set to $-3^{\circ} \mathrm{C}$ with the bottom set to $-18^{\circ} \mathrm{C}$. The SAA translation speed was $10 \mathrm{microns} / \mathrm{sec}$; the solid/liquid interface is seen in Figure 24. 


\section{Discussion}

The MMaJIC ground demonstration unit has shown successful temperature control, data acquisition, and the ability to accommodate a wide range of solidification experiments. The simplicity of MMaJIC and the short lead-time of sample tray fabrication can offer a path to conducting many experiments in a short time with minimum expense aboard the ISS. Updates include reducing noise, installing a camera, filtering EMF interference in data acquisition, and creating novel trays. With these reforms, MMaJIC can further minimize crew time, improve testing and data acquisition, and offer new experimental venues outside of solidification studies. These could include combustion experiments, fluids experiments, and particle motion studies. Again, the sample trays design is highly flexible and can be fabricated to accommodate any number of novel experiments. SoLIDD was partially functional and offers the potential of conducting controlled directional solidification experiments aboard the ISS. Like MMaJIC, SoLIDD uses minimum crew interaction and time. The ISS astronaut would insert the sample, set the desired cooler and heater temperatures, set the speed of the actuator, and press start. Once SoLIDD is aboard the ISS, samples can be continually supplied to accommodate several investigations. ICED-T developed into a fully functioning, breadboard, unit that successfully demonstrated establishing a solid/liquid interface in transparent low-melting point liquids such as water. Once established, the solid/liquid interface can be slowly moved at 1 micron/sec which provides key insight regarding the microstructural development. Like MMaJIC and SoLIDD, ICED-T is conceptually simple to use. It does, however, require command from the ground for real-time experimental parameter changes and therefore must be connected with an ISS laptop.

\section{Acknowledgments}

The SoLIDD and MMaJIC team would like to thank Dr. Richard Grugel (EM-31) for his support as a mentor, Jeff Quick (EM-31) for machining some components in SoLIDD and MMaJIC, Ellen Rabenberg (EM-31) for part orders, Taylor Waddell and Natasha Liston-Beck (ES-62) for the assistance and access to the Makerspace in laser cutting and 3D printing, Dr. Louise Strutzenberg (ER-42) for her assistance, Carey Harris for engineering assistance, and Luke Scharber (ER-41) provided electronics parts and Arduinos. The team made use of the Arduino PID library. The team wanted to thank the NASA Marshall Space Flight Center and the Wisconsin Space Grant Consortium for the funding internships and the opportunity to work on MMaJIC and SoLIDD.

\section{References}

Costello, K., Ferguson, S., and Kinney, S. (2014). Guidelines for Risk Management. Version F, NASA Independent Verification \& Validation Program.

Daly, Shannen, (2017). MMaJIC, an Experimental Chamber for Investigating Soldering and Brazing in Microgravity. Gravitational and Space Research, vol. 5 no. 2, 31, pp. 28-34.

McElderry, J., Phillips T., Hardyman M., Ragan J., and Rameshbabu R. (2018). NASA Microgravity Solidification Experiments for the International Space Station. Proceedings of the Wisconsin Space Conference, vol. 1, no. 1.

National Aeronautics and Space Administration, Lyndon B. Johnson Space Center. (1998). International Space Station Familiarization. Houston, Texas: National Aeronautics and Space Administration.

Spivey, R., Gilley, S., Ostrogorsky, A., Grugel, R., and Smith, G. (2003). SUBSA and PFMI Transparent Furnace Systems Currently in use in the International Space Station Microgravity Science Glovebox. AIAA 20031362. 41th AIAA Aerospace Sciences Meeting \& Exhibit. 